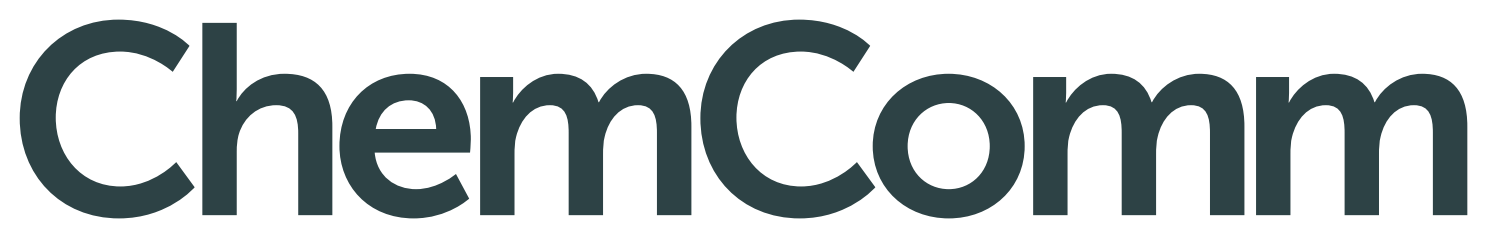

Chemical Communications rsc.li/chemcomm

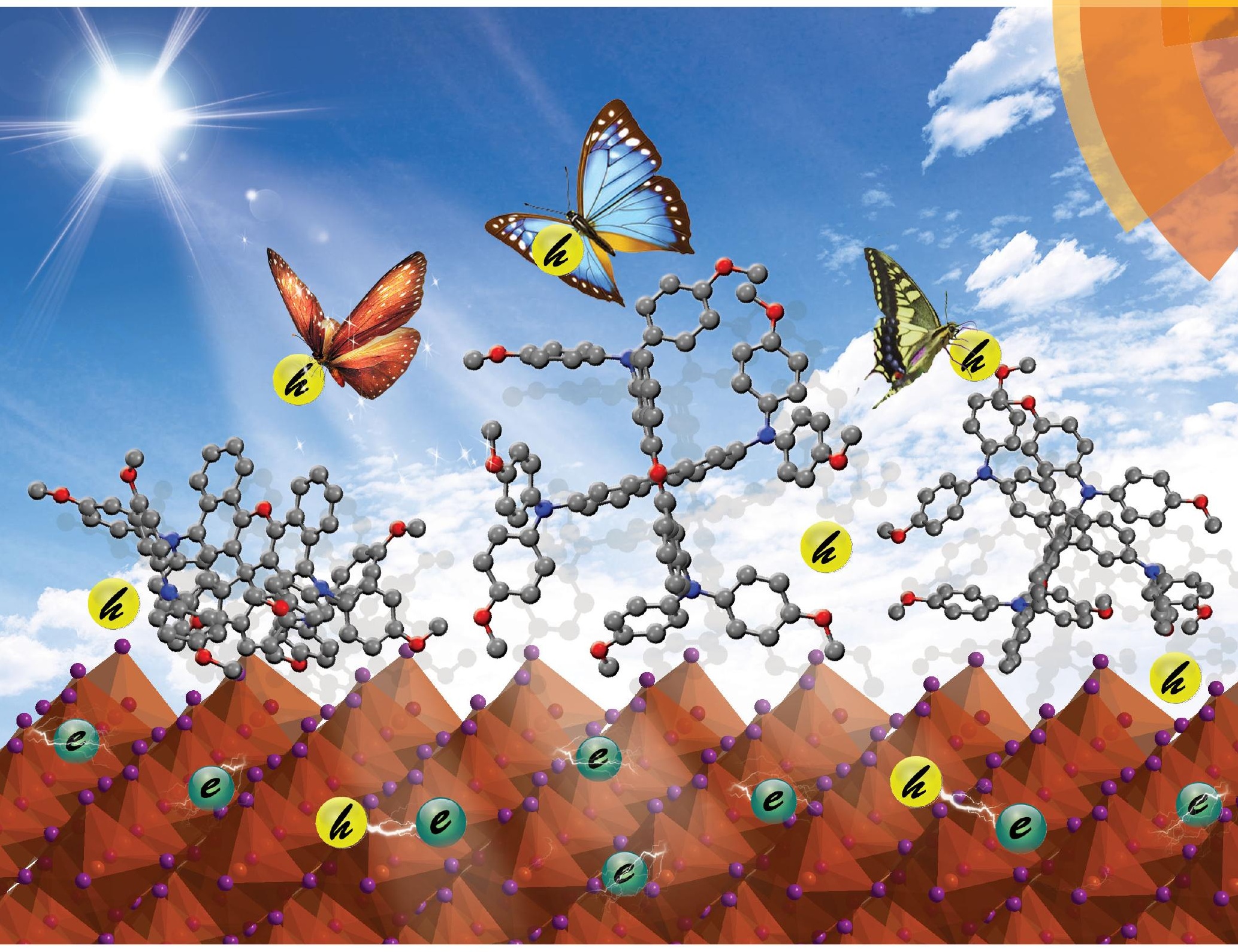

ISSN 1359-7345

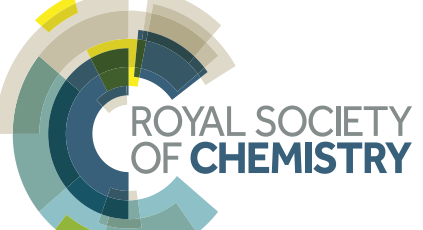


Check for updates

Cite this: Chem. Commun., 2018, 54,9571

Received 21st May 2018, Accepted 13th July 2018

DOI: $10.1039 / \mathrm{c} 8 \mathrm{cc} 04026 \mathrm{e}$

rsc.li/chemcomm

\section{Design and synthesis of dopant-free organic hole-transport materials for perovskite solar cells $\dagger$}

\author{
Linqin Wang, $\ddagger^{\mathrm{a}}$ Jinbao Zhang, $\ddagger^{\mathrm{b}}$ Peng Liu, $\ddagger^{\mathrm{c}}$ Bo Xu, ${ }^{\mathrm{d}}$ Biaobiao Zhang, (D) ${ }^{\mathrm{a}}$ \\ Hong Chen, (D) A. Ken Inge, (DD) Yuanyuan Li, (D) Haoxin Wang, ${ }^{\mathrm{g}}$ \\ Yi-Bing Cheng, (D) ${ }^{b}$ Lars Kloo $^{c}$ and Licheng Sun (D)*ag
}

Two novel dopant-free hole-transport materials (HTMs) with spiro[dibenzo[c,h]xanthene-7,9'-fluorene] (SDBXF) skeletons were prepared via facile synthesis routes. A power conversion efficiency of $15.9 \%$ in perovskite solar cells is attained by using one HTM without dopants, which is much higher than undoped Spiro-OMeTAD-based devices (10.8\%). The crystal structures of both new HTMs were systematically investigated to reveal the reasons behind such differences in performance and to indicate the design principles of more advanced HTMs.

Perovskite solar cells (PSCs) have attracted great attention in the past few years with a certified power conversion efficiency (PCE) up to $22.7 \% .{ }^{1-5}$ In PSC devices, novel hole-transport materials (HTMs) play a critical role for the improvement of device performance and stability. ${ }^{6}$ The most well-known HTM, Spiro-OMeTAD $\left(2,2^{\prime}, 7,7^{\prime}\right.$-tetrakis( $N, N^{\prime}$-di- $p$-methoxyphenylamine)$9,9^{\prime}$-spirobifluorene), was originally reported by Grätzel and co-workers. ${ }^{7}$ However, the high cost of the raw materials and the tedious synthetic routes limit its wide application. Besides, organic HTMs typically show relatively low conductivity, and therefore most of the reported HTMs need to be doped with additives, in order to increase the conductivity of the HTM and

\footnotetext{
${ }^{a}$ Organic Chemistry, Department of Chemistry, KTH Royal Institute of Technology, SE-10044 Stockholm, Sweden. E-mail: lichengs@kth.se

${ }^{b}$ Department of Materials Science and Engineering, 22 Alliance Lane, Monash University, VIC 3800, Australia

${ }^{c}$ Applied Physical Chemistry, Center of Molecular Devices, Department of Chemistry, KTH-Royal Institute of Technology, SE-10044 Stockholm, Sweden

${ }^{d}$ Department of Chemistry, Ångström Laboratory, Box 523, Uppsala University, 75120 Uppsala, Sweden

${ }^{e}$ Department of Material and Environmental Chemistry (MMK), Stockholm University, SE-10691 Stockholm, Sweden

${ }^{f}$ Wallenberg Wood Science Center, Department of Fibre and Polymer Technology, KTH Royal Institute of Technology, SE-100 44 Stockholm, Sweden

${ }^{g}$ State Key Laboratory of Fine Chemicals, Institute of Artificial Photosynthesis, DUT-KTH Joint Education and Research Centre on Molecular Devices,

Dalian University of Technology, 116024 Dalian, China

$\dagger$ Electronic supplementary information (ESI) available. CCDC 1844277 and 1844276. For ESI and crystallographic data in CIF or other electronic format see DOI: $10.1039 / \mathrm{c} 8 \mathrm{cc} 04026 \mathrm{e}$

\$ These authors contribute equally to this work.
}

the PCE of the PSCs. ${ }^{8-10}$ The dopants, however, are commonly hydrophilic, which have been shown to accelerate the degradation of the perovskite crystals and induce device instability. Therefore, the design of novel dopant-free HTMs is necessary for applications in PSCs. Efforts have been devoted to develop new dopant-free HTMs based on different core structures. ${ }^{11-14}$ For example, Xu et al. recently designed and synthesized two HTMs based on the indacenodithiophene (IDT) unit with long alkyl chains as substituents. ${ }^{15}$ The co-planarity of the IDT units was found to improve intermolecular interactions and most likely promote a higher hole mobility with methoxytriphenylamine (TPA) as the extending units. Sun and Johansson et al. reported a dopant-free HTM including the charged moieties accompanied by counter ions. ${ }^{16}$ They found that the new type of ionic HTM shows a higher hole conductivity, and the obtained devices demonstrated better PCE than standard Spiro-OMeTAD-based devices. Jen and Chen et al. presented a new structural HTM, Trux-OMeTAD, consisting of a $C_{3 \mathrm{~h}}$ truxene core with arylamine terminals and hexyl side-chains. ${ }^{17}$ The PSCs based on Trux-OMeTAD as the HTM showed a high PCE of $18.6 \%$ with minimal hysteresis. In addition, excellent hole mobility was exhibited in this planar, rigid and fully conjugated molecule, and suitable energy levels matched those of the perovskite materials.

Recently, several HTMs were reported with a spiro[fluorine9, $9^{\prime}$-xanthene] (SFX) backbone as the core unit. ${ }^{18-20}$ In the SFX unit, there is a tetrahedral $\mathrm{sp}^{3}$-hybridized carbon atom between two perpendicular $\pi$ systems composing a three-dimensional (3D) structural architecture which can reduce both charge recombination and electronic coupling in the HTM layer. In addition, this rigid spiro conjugation is able to enhance the charge-transport ability of hole transfer. ${ }^{21}$ Previous work from our group showed that the estimated synthetic cost for the SFX core is 30 times lower than that of the spiro-bi[fluorine] (SBF) core, and a synthesis yield of over $90 \%$ can be achieved when phenol and 9-fluorenone are used as raw materials. ${ }^{10,22}$

One of the more promising strategies to construct dopant-free HTMs for PSCs is based on the introduction of larger $\pi$-conjugated systems to the HTM molecules, which have the potential to 
improve the conductivity of the hole transport layers. Herein, two new dopant-free HTMs, $N^{2^{\prime}}, N^{2^{\prime}}, N^{7^{\prime}}, N^{7^{\prime}}$-tetrakis(4-methoxyphenyl)spiro[dibenzo[ $c, h]$ xanthene-7, $9^{\prime}$-fluorene]-2' ${ }^{\prime} 7^{\prime}$-diamine (X61) and $N^{2^{\prime}}, N^{2^{\prime}}, N^{5}, N^{5}, N^{7^{\prime}}, N^{7^{\prime}}, N^{9}, N^{9}$-octakis(4-methoxyphenyl)spiro[dibenzo$[c, h]$ xanthene-7, $9^{\prime}$-fluorene]-2', ${ }^{\prime}, 7^{\prime}, 9$-tetraamine (X62), with spiro[dibenzo[c, $h]$ xanthene-7,9'-fluorene] (SDBXF) as the skeleton were designed and synthesized. A systematic crystallographic investigation of both HTMs offers more detailed insights into the molecular packing features. The SDBXF skeleton also represents a spiro core but has an extended $\pi$ conjugation system in comparison to the well-known SFX skeleton. The introduction of the naphthalene group could increase the conjugation system of the HTMs, leading to more efficient $\pi-\pi$ stacking in HTM films. This is expected to be beneficial for the charge transport.

The molecular structures obtained from single crystal X-ray diffraction (SCXRD) of the two HTMs are shown in Fig. 1. In our approach to synthesize the final products X61 and X62, 1-naphthol and 2,7-dibromo-9-fluorenone were used as starting materials, respectively, followed by a simple Buchwald-Hartwig reaction. Detailed experimental procedures of the synthesis are presented in the ESI. $\dagger$ The normalized UV-visible absorption and photoluminescence spectra of X61 and X62 are shown in Fig. S7 (ESI $\dagger$ ), and the corresponding data are listed in Table S1 (ESI $\dagger$ ). Both X61 and X62 display similar maximum absorption peaks around $382 \mathrm{~nm}$. Their narrow absorption spectra below $430 \mathrm{~nm}$ are favourable for minimizing parasitic absorption from the HTM layer for utilization in perovskite tandem solar cell devices. The emission peaks of X61 and X62 at $432 \mathrm{~nm}$ and $483 \mathrm{~nm}$, respectively, indicate that the dimethoxydiphenylamine units induce only small effects on the absorption properties but apparently affect the emission properties. The optical band gap $E_{0-0}$ of the HTMs can be obtained from the intersection of the absorption and emission spectra, which are shown in Table S1 (ESI $\dagger$ ).

In order to investigate their redox properties, the oxidation potentials of both HTMs were also studied using cyclic voltammetry

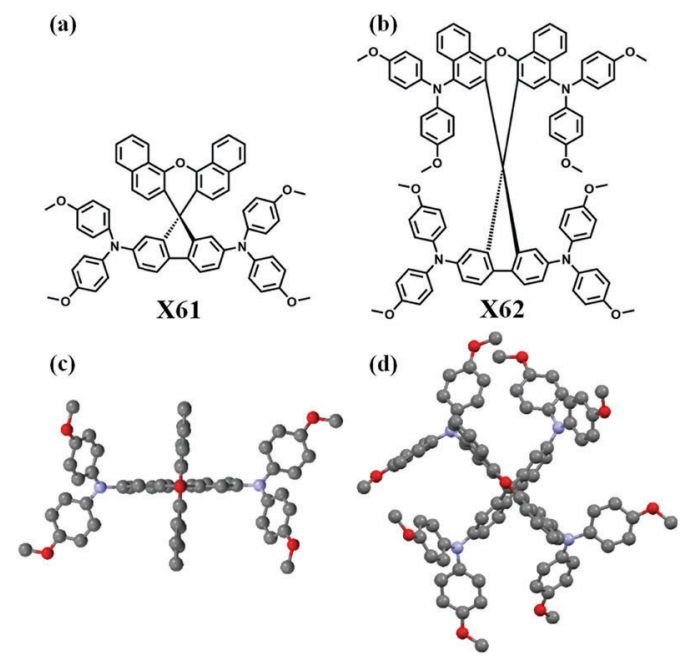

Fig. 1 Molecular structures and single crystal X-ray structures of HTM X61 ( $a$ and $c$ ) and X62 (b and d).

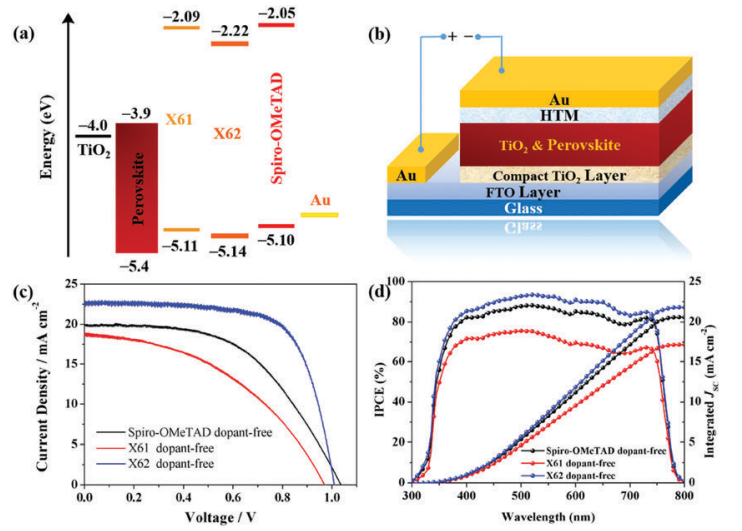

Fig. 2 (a) Energy level diagram; (b) sketch map of the PSCs; (c) J-V characteristics of X61, X62, and Spiro-OMeTAD-based PSCs measured under $100 \mathrm{~mW} \mathrm{~cm}{ }^{-2}$ AM 1.5G; (d) the corresponding IPCE spectra and integrated $J_{S C}$ values of the devices.

(CV) and differential pulsed voltammetry (DPV) (Fig. S8 and S9, ESI, $\uparrow$ respectively) in DCM solution with a concentration of $10^{-4} \mathrm{M}$, and the corresponding data are listed in Table S1 (ESI $\dagger$ ). The highest occupied molecular orbital (HOMO) energy levels of the HTMs were determined via the above procedure. Both HTMs show a similar HOMO energy level $(-5.11 \mathrm{eV}$ for $\mathbf{X 6 1}$ and $-5.14 \mathrm{eV}$ for X62) as that of Spiro-OMeTAD $(-5.10 \mathrm{eV})$. This indicates that both molecules are suitable for hole transfer processes from the perovskite layer to the HTM films, and match the work function of the Au counter electrode. For the lowest unoccupied molecular orbital (LUMO) energy levels, as shown in Fig. 2a, both HTMs have much higher LUMO energy levels $(-2.09 \mathrm{eV}$ and $-2.22 \mathrm{eV}$ for X61 and X62, respectively) than the conduction band (CB) of the perovskite, suggesting that the transfer of the photogenerated electrons from the perovskite layer to the Au counter electrode material is effectively suppressed. For a better understanding of the energy levels and the electronic distribution in the molecular building blocks in both HTMs, density functional theory (DFT) calculations were performed at the B3LYP/6-31G(d) level with the Gaussian 09 program (see Fig. S10, ESI $\dagger$ ). The HOMOs of both molecules are delocalized in the bottom fluorene and dimethoxydiphenylamine units, while the LUMOs of these molecules are mainly delocalized in the dibenzo-xanthene moieties. The spatially separated HOMOs and LOMOs could forecast good hole transporting properties.

As mentioned before, charge transport is a significant factor to consider in the design of highly efficient HTMs for PSCs. Both the hole mobility and conductivity are critical factors for determining the potential eligibility of new HTMs in PSCs, since they can significantly influence the hole collection efficiency in the devices. In this work, we used two-probe electrical conductivity measurements to investigate the conductivities of the HTMs and the space charge limited current (SCLC) method to determine the hole mobility. The obtained $J-V$ curves are shown in Fig. S11 and S12 (ESI $\dagger$ ), and the corresponding data are estimated as shown in Table S2 (ESI $\dagger$ ). The HTM solutions used for film formation in the above measurements were the same as those used when manufacturing the solar cell devices 
without dopants, and all details can be found in the ESI. $\dagger$ Under such conditions, X62 shows a higher conductivity $(5.14 \times$ $\left.10^{-6} \mathrm{~S} \mathrm{~cm}^{-1}\right)$ than $\mathbf{X 6 1}\left(3.98 \times 10^{-6} \mathrm{~S} \mathrm{~cm}^{-1}\right)$ and Spiro-OMeTAD $\left(4.56 \times 10^{-6} \mathrm{~S} \mathrm{~cm}^{-1}\right)$. This may be due to X62 having a larger conjugated system and structural extension, resulting in more efficient $\pi-\pi$ stacking in the hole transporting films. In addition, X62 also exhibits a higher hole mobility $\left(7.95 \times 10^{-5} \mathrm{~cm}^{2} \mathrm{~V}^{-1} \mathrm{~s}^{-1}\right)$ than X61 $\left(3.91 \times 10^{-5} \mathrm{~cm}^{2} \mathrm{~V}^{-1} \mathrm{~s}^{-1}\right)$ and Spiro-OMeTAD $(6.06 \times$ $\left.10^{-5} \mathrm{~cm}^{2} \mathrm{~V}^{-1} \mathrm{~s}^{-1}\right)$.

The PSC devices were fabricated with the standard structure of $\mathrm{FTO} / \mathrm{TiO}_{2}$ compact layer/meso-TiO $/$ /mixed-perovskite/HTM/Au. The concentration of the HTM solutions used for film formation by spin coating in the devices was optimized to $18 \mathrm{mg} \mathrm{mL}^{-1}$. In addition, the HTM coverage on top of the perovskite layer was investigated using scanning electron microscopy (SEM) (Fig. S13, $\mathrm{ESI} \dagger$ ), from which we noted that the perovskite crystal was fully covered by the HTM materials when the solution concentration exceeds $15 \mathrm{mg} \mathrm{mL}^{-1}$. Obviously, X62 shows a better film-forming ability that can effectively suppress charge recombination between the perovskite and the HTM, which is coherent with the recombination resistance $\left(R_{\text {rec}}\right)$ obtained from electrochemical impedance spectroscopy (EIS, Fig. S21 and Table S4, ESI $\dagger$ ). Fig. 2c shows the $J-V$ curves of the PSCs based on different HTMs and the corresponding parameters are given in Table 1. Under optimal conditions, the X62-based PSCs showed the best performance with a PCE of $15.9 \%$, a shortcircuit photocurrent density $\left(J_{\mathrm{SC}}\right)$ of $22.4 \mathrm{~mA} \mathrm{~cm}^{-2}$, an open-circuit photovoltage $\left(V_{\mathrm{OC}}\right)$ of $1.01 \mathrm{~V}$, and a fill factor $(\mathrm{FF})$ of $70.4 \%$. In contrast, the X61-based PSC devices yielded a lower PCE of $8.0 \%$, with a $J_{\mathrm{SC}}$ of $18.6 \mathrm{~mA} \mathrm{~cm} \mathrm{~cm}^{-2}$, a $V_{\mathrm{OC}}$ of $0.97 \mathrm{~V}$, and an $\mathrm{FF}$ of $44.1 \%$. As a reference, devices based on the standard HTM Spiro-OMeTAD under the same conditions showed an efficiency of $10.8 \%$, a $J_{\mathrm{SC}}$ of $19.9 \mathrm{~mA} \mathrm{~cm}^{-2}$, a $V_{\mathrm{OC}}$ of $1.04 \mathrm{~V}$ and an FF of $52.6 \%$. Fig. $2 \mathrm{~d}$ shows the incident photon-to-current-conversion efficiency (IPCE) spectra and the corresponding integrated $J_{\mathrm{SC}}$ of the PSC devices with X61, X62 and Spiro-OMeTAD. The X62-based PSC devices show much higher IPCE values than those based on both X61 and SpiroOMeTAD. The calculated photocurrents from the IPCE spectra agree well with the $J_{\text {sc }}$ values obtained from the $J-V$ experiments.

Among these devices, the X62-based PSCs reached the highest $J_{\mathrm{SC}}$, which might be due to a higher charge collection efficiency ascribed to the higher hole mobility and conductivity. As a comparison, the lower hole conductivity of X61 and SpiroOMeTAD results in a higher series resistance in the devices, which is in good agreement with the series resistance $\left(R_{\mathrm{s}}\right)$ determined via EIS and shown in Fig. S21 and Table S4 (ESI $\dagger$ ). Besides, the higher resistance of the $\mathbf{X 6 1}$ and Spiro-OMeTAD

Table 1 Photovoltaic parameters of the devices based on the dopant-free HTMs X61, X62 and Spiro-OMeTAD

\begin{tabular}{llllll}
\hline HTMs & $\begin{array}{l}J_{\text {SC }} \\
\left(\mathrm{mA} \mathrm{cm} \mathrm{cm}^{-2}\right)\end{array}$ & $\begin{array}{l}J_{\text {SC Cal. }} \\
\left(\mathrm{mA} \mathrm{cm}^{-2}\right)\end{array}$ & $V_{\text {OC }}(\mathrm{V})$ & FF $(\%)$ & PCE (\%) \\
\hline X61 & 18.6 & 17.2 & 0.97 & 44.1 & 8.0 \\
X62 & 22.4 & 21.9 & 1.01 & 70.4 & 15.9 \\
Spiro-OMeTAD & 19.9 & 20.6 & 1.04 & 52.6 & 10.8
\end{tabular}

films leads to lower FF values in the PSC devices. However, the $V_{\text {OC }}$ values of both X61 and X62 based devices are lower than that of Spiro-OMeTAD-based PSCs, probably due to a higher recombination loss in the two new materials. The device stability was investigated under ambient conditions at room temperature with a relative humidity of about $50 \%$. After 10 days, the efficiencies of the undoped Spiro-OMeTAD, X61 and X62 based solar cells retained $81 \%, 49 \%$ and $80 \%$ of the initial efficiency, respectively (Fig. S20, ESI $\dagger$ ). This result demonstrates that dopant-free HTMs play a critical role in the long-term stability of the PSCs. The enhanced device stability of $\mathbf{X 6 2}$ is another promising property for its future application in PSCs.

In order to further investigate the photovoltaic properties of the PSCs based on X61 and X62, the hole transfer kinetics at the perovskite/HTM interface were characterized via steady-state photoluminescence and time-resolved fluorescence decay (Fig. S14, $\mathrm{ESI} \dagger$ ). The charge transfer occurring at the perovskite/HTM interface is shown in Fig. S14a (ESI $\dagger$ ). The pristine perovskite film shows a long-lived decay with a half time constant of $85 \mathrm{~ns}$, while the perovskite films capped by the HTMs exhibit a much shorter time constant. The $\mathbf{X 6 1}$ coated perovskite film shows a half time constant of $7.6 \mathrm{~ns}$. In comparison, the $\mathbf{X 6 2}$ coated one exhibits the shortest half time constant of $7.1 \mathrm{~ns}$. This indicates that $\mathbf{X 6 2}$ mediates a faster hole-transport process, which agrees well with the higher $J_{\mathrm{SC}}$ obtained from the X62-based PSCs.

In order to gain further insights into the charge transport of these two SDBXF skeleton based HTMs, single crystals of X61 and $\mathbf{X 6 2}$ were grown via a liquid diffusion method from organic solvents. The crystal structures of $\mathbf{X 6 1}$ (triclinic, space group: $P \overline{1}$ ) and $\mathbf{X 6 2}$ (monoclinic, space group: $P 2_{1} / c$ ) were determined via single crystal X-ray diffraction (SCXRD) analysis, as shown in Fig. S15 (ESI $\dagger$ ), and the perspective views along different unit cell axes are illustrated in Fig. S16 (ESI $\dagger$ ).

The dihedral angles between the dibenzo[c,h]xanthene (DBX) unit and the fluorene unit are $89.6^{\circ}$ and $89.2^{\circ}$, respectively, for X61 and X62 (see Fig. 3a and b), which are similar to that in Spiro-OMeTAD $\left(89.9^{\circ}\right){ }^{23}$ These dihedral angles indicate weak

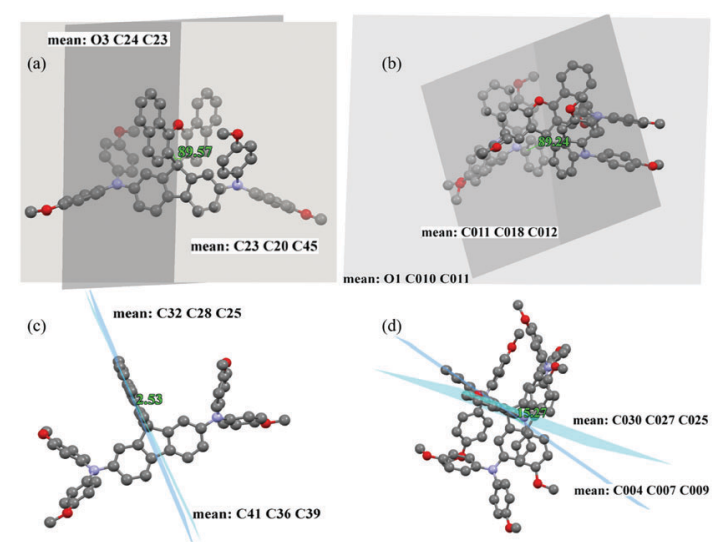

Fig. 3 Dihedral angles of X61 ( $a$ and $c$ ) and X62 ( $b$ and d) obtained from SCXRD. The light grey, dark grey, light blue and dark blue colored planes represent the dibenzo[c,h]xanthene unit, fluorene unit and two naphthalene units, respectively. Hydrogen atoms are omitted for clarity. 

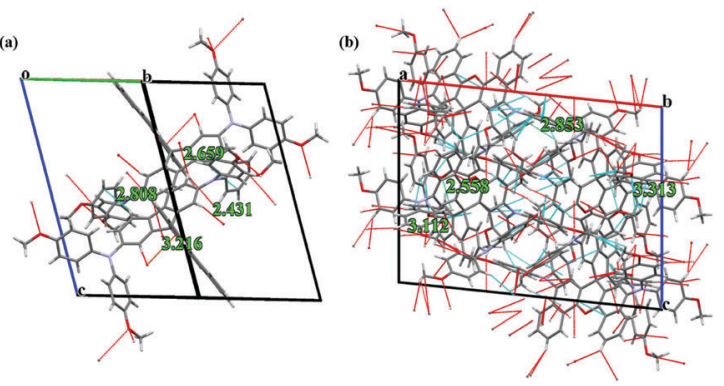

Fig. $4 \mathrm{O}-\mathrm{H}, \mathrm{CH} / \pi$ hydrogen bonds, $\mathrm{C}-\mathrm{O}$, and $\pi-\pi$ short contacts observed in the packing of X61 (a) and X62 (b) crystals obtained from SCXRD.

intermolecular interactions with minimal molecular stacking which benefit the formation of amorphous solids. ${ }^{24}$ The angles between the two close naphthalene units next to the non-aromatic central ring are $2.5^{\circ}$ and $15.3^{\circ}$, respectively, for X61 and X62 (see Fig. $3 \mathrm{c}$ and $\mathrm{d}$ ), which may weaken the $\pi-\pi$ interaction between any two DBX units preventing dense packing and aggregation. The crystal packing details of X61 and X62 are provided in Fig. S17 (ESI $\dagger$ ).

In X61 (Fig. 4a), the shortest $\pi-\pi$ contact between the DBX unit and the dimethoxyphenylamine moieties is $3.216 \AA$, while the interaction distances are $2.808 \AA$ and $2.659 \AA$ for $\mathrm{CH} / \pi$ interactions and $\mathrm{O}-\mathrm{H}$ hydrogen bonds, respectively. As for X62 (Fig. 4b), when observed perpendicular to the $a c$ plane (i.e. view along the $b$ axis), each molecule has multiple short contacts with the surrounding molecules, including $\mathrm{O}-\mathrm{H}, \mathrm{CH} / \pi$ hydrogen bonds, $\mathrm{C}-\mathrm{O}$, and $\pi-\pi$ short contacts with the shortest interaction distances of $2.567 \AA, 2.882 \AA$, $3.112 \AA$ and $3.313 \AA$. The large abundance of short contacts between the $\mathbf{X 6 2}$ molecules could provide sufficient channels for efficient charge transport and result in better photovoltaic performance. Therefore, incorporation of suitable substituents on the dibenzo $[c, h]$ xanthene skeleton is of great importance for $3 \mathrm{D}$ spatial extension in molecular stacking and for enhancing the charge transporting properties of new HTMs.

In summary, we have successfully designed and synthesized two new spiro-type HTMs, named X61 and X62, with SDBXF as the skeleton. X-ray crystallographic analysis of the single crystals of both HTMs was carried out in this study, in which multiple short contacts are found to be beneficial for the enhancement of charge transfer. The corresponding dopant-free PSCs based on X62 exhibit a maximum efficiency of $15.96 \%$ (working area, $0.16 \mathrm{~cm}^{2}$ ), which outperform the standard Spiro-OMeTAD based devices (10.84\%). A higher IPCE value shown by devices based on $\mathbf{X 6 2}$ is consistent with the high $J_{\mathrm{SC}}$ of $22.42 \mathrm{~mA} \mathrm{~cm}{ }^{-2}$. Our study highlights the potential application of SDBXF-skeleton-based molecules as dopant-free HTMs for high performance PSCs.

The authors would like to thank the Swedish Energy Agency, SSF and the China Scholarship Council (CSC) for financial support. We also greatly thank Dr Zhaoyang Yao, Dr Fuguo Zhang, Dr Ming Cheng and Dr Yong Hua (KTH) for their helpful discussions. Dr A. Ken Inge thanks KAW and SSF for financial support. YBC acknowledges the support of the Australia Research Council Centre of Excellence in Exciton Science (project number CE 170100026).

\section{Conflicts of interest}

There are no conflicts to declare.

\section{References}

1 M. M. Lee, J. Teuscher, T. Miyasaka, T. N. Murakami and H. J. Snaith, Science, 2012, 338, 643-647.

2 M. Liu, M. B. Johnston and H. J. Snaith, Nature, 2013, 501, 395-398.

3 D. Bi, W. Tress, M. I. Dar, P. Gao, J. Luo, C. Renevier, K. Schenk, A. Abate, F. Giordano, J. P. Correa Baena, J. D. Decoppet, S. M. Zakeeruddin, M. K. Nazeeruddin, M. Gratzel and A. Hagfeldt, Sci. Adv., 2016, 2, e1501170.

4 M. Saliba, T. Matsui, K. Domanski, J. Y. Seo, A. Ummadisingu, S. M. Zakeeruddin, J. P. Correa-Baena, W. R. Tress, A. Abate, A. Hagfeldt and M. Gratzel, Science, 2016, 354, 206-209.

5 https://www.nrel.gov/pv/assets/images/efficiency-chart.png.

6 L. Calio, S. Kazim, M. Gratzel and S. Ahmad, Angew. Chem., Int. Ed. Engl., 2016, 55, 14522-14545.

7 M. Grätzel, U. Bach, D. Lupo, P. Comte, J. E. Moser, F. Weissörtel, J. Salbeck and H. Spreitzer, Nature, 1998, 395, 583-585.

8 J. Zhang, Y. Hua, B. Xu, L. Yang, P. Liu, M. B. Johansson, N. Vlachopoulos, L. Kloo, G. Boschloo, E. M. J. Johansson, L. Sun and A. Hagfeldt, Adv. Energy Mater., 2016, 6, 1601062.

9 W. H. Nguyen, C. D. Bailie, E. L. Unger and M. D. McGehee, J. Am. Chem. Soc., 2014, 136, 10996-11001.

10 B. Xu, J. Zhang, Y. Hua, P. Liu, L. Wang, C. Ruan, Y. Li, G. Boschloo, E. M. J. Johansson, L. Kloo, A. Hagfeldt, A. K. Y. Jen and L. Sun, Chem, 2017, 2, 676-687.

11 W. S. Yang, B. W. Park, E. H. Jung, N. J. Jeon, Y. C. Kim, D. U. Lee, S. S. Shin, J. Seo, E. K. Kim, J. H. Noh and S. I. Seok, Science, 2017, 356, 1376-1379.

12 G.-W. Kim, G. Kang, J. Kim, G.-Y. Lee, H. I. Kim, L. Pyeon, J. Lee and T. Park, Energy Environ. Sci., 2016, 9, 2326-2333.

13 J. Liu, Y. Wu, C. Qin, X. Yang, T. Yasuda, A. Islam, K. Zhang, W. Peng, W. Chen and L. Han, Energy Environ. Sci., 2014, 7, 2963-2967.

14 H.-C. Liao, T. L. D. Tam, P. Guo, Y. Wu, E. F. Manley, W. Huang, N. Zhou, C. M. M. Soe, B. Wang, M. R. Wasielewski, L. X. Chen, M. G. Kanatzidis, A. Facchetti, R. P. H. Chang and T. J. Marks, Adv. Energy Mater., 2016, 6, 1600502.

15 X. Liu, E. Rezaee, H. Shan, J. Xu, Y. Zhang, Y. Feng, J. Dai, Z.-K. Chen, W. Huang and Z.-X. Xu, J. Mater. Chem. C, 2018, 6, 4706-4713.

16 J. Zhang, B. Xu, L. Yang, A. Mingorance, C. Ruan, Y. Hua, L. Wang, N. Vlachopoulos, M. Lira-Cantú, G. Boschloo, A. Hagfeldt, L. Sun and E. M. J. Johansson, Adv. Energy Mater., 2017, 7, 1602736.

17 C. Huang, W. Fu, C. Z. Li, Z. Zhang, W. Qiu, M. Shi, P. Heremans, A. K. Jen and H. Chen, J. Am. Chem. Soc., 2016, 138, 2528-2531.

18 B. Xu, Z. Zhu, J. Zhang, H. Liu, C.-C. Chueh, X. Li and A. K. Y. Jen, Adv. Energy Mater., 2017, 7, 1700683.

19 G. Kadam, A. Anuradha, A. Agarwal, A. Puyad, D. D. La, R. A. Evans, J. Li, A. Gupta and S. V. Bhosale, Mater. Chem. Front., 2018, 2, 1090.

20 M. Maciejczyk, A. Ivaturi and N. Robertson, J. Mater. Chem. A, 2016, 4, 4855-4863.

21 V. Govindan, K.-C. Yang, Y.-S. Fu and C.-G. Wu, New J. Chem., 2018, 42, 7332-7339.

22 B. Xu, D. Bi, Y. Hua, P. Liu, M. Cheng, M. Grätzel, L. Kloo, A. Hagfeldt and L. Sun, Energy Environ. Sci., 2016, 9, 873-877.

23 P. Ganesan, K. Fu, P. Gao, I. Raabe, K. Schenk, R. Scopelliti, J. Luo, L. H. Wong, M. Grätzel and M. K. Nazeeruddin, Energy Environ. Sci., 2015, 8, 1986-1991.

24 D. Bi, B. Xu, P. Gao, L. Sun, M. Grätzel and A. Hagfeldt, Nano Energy, 2016, 23, 138-144. 